Open Access

\title{
Patient-Reported Outcomes Measurement Information System (PROMIS) instruments among individuals with symptomatic knee osteoarthritis: a cross-sectional study of floor/ceiling effects and construct validity
}

\author{
Jeffrey B. Driban ${ }^{1 *}$, Nani Morgan ${ }^{1,2}$, Lori Lyn Price ${ }^{3}$, Karon F. Cook ${ }^{4}$ and Chenchen Wang ${ }^{1}$
}

\begin{abstract}
Background: The psychometric properties of Patient Reported Outcomes Measurement Information System (PROMIS) instruments have been explored in a number of general and clinical samples. No study, however, has evaluated the psychometric function of these measures in individuals with symptomatic knee osteoarthritis (KOA). The aim of this project was to evaluate the construct (structural) validity and floor/ceiling effects of four PROMIS measures in this population.

Methods: We conducted a secondary analysis of baseline data from a randomized trial comparing Tai Chi and physical therapy. Participants completed four PROMIS static short-form instruments (i.e., Anxiety, Depression, Physical Function, and Pain Interference) as well as six well-validated (legacy) measures that assess pain, function, and psychological health. We calculated descriptive statistics and percentages of participants scoring the minimum (floor) and maximum (ceiling) possible scores for PROMIS and legacy measures. We also estimated the association between PROMIS scores and scores on legacy measures using Spearman's rank correlations coefficients.
\end{abstract}

Results: Data from 204 participants were analyzed. Mean age of the sample was 60 years; $70 \%$ were female. The PROMIS Anxiety and Depression had floor effects with 17 and $24 \%$ of participants scoring the minimum, respectively. PROMIS Anxiety and Depression scores had strongest associations with general mental health, including stress (Perceived Stress Scale, $r \geq 0.65$ ) and depression (Beck Depression Index-II, $r=0.70$ ). PROMIS Pain Interference scores correlated most strongly with measures of whole body pain (Short-Form 36 Bodily Pain, $r=-0.73$ ) and physical health (Short-Form 36 Physical-Component Summary, $r=-0.73$ ); their correlations were lower with other legacy measures, including with the WOMAC knee-specific pain $(r=0.47)$. PROMIS Physical Function scores had stronger associations with scores on the Short-Form 36 Physical Function $(r=0.79)$ than with scores on other legacy measures.

Conclusion: The four PROMIS static-short forms performed well among individuals with symptomatic knee osteoarthritis as evidenced in correlations with legacy measures. PROMIS Anxiety and Depression target general mental health (e.g., stress, depression), and PROMIS Pain Interference and Physical Function static-short forms target whole-body outcomes among participants with symptomatic knee osteoarthritis. Floor effects in the PROMIS Anxiety and Depression scores should be considered if needing to distinguish among patients with very low levels of these outcomes.

Trial registration: Clinicaltrials.gov NCT01258985. Registered 10 December 2010

\footnotetext{
* Correspondence: jeffrey.driban@tufts.edu

'Division of Rheumatology, Tufts Medical Center, 800 Washington Street, Box \#406, Boston, MA 02111, USA

Full list of author information is available at the end of the article
}

\section{Biomed Central}

(c) 2015 Driban et al. Open Access This article is distributed under the terms of the Creative Commons Attribution 4.0 International License (http://creativecommons.org/licenses/by/4.0/), which permits unrestricted use, distribution, and reproduction in any medium, provided you give appropriate credit to the original author(s) and the source, provide a link to the Creative Commons license, and indicate if changes were made. The Creative Commons Public Domain Dedication waiver (http://creativecommons.org/publicdomain/zero/1.0/) applies to the data made available in this article, unless otherwise stated. 


\section{Background}

Osteoarthritis is a disease characterized by structural changes throughout the joint but it is also an illness of the whole patient defined by patient-reported outcomes such as pain, stiffness, and disability [1]. Furthermore, a patient with osteoarthritis often participates less in valued activities and may experience fatigue as well as disrupted mood, sleep, and quality of life [2-4]. Therefore, we must measure a wide array of patient-reported outcomes to thoroughly assess health status and treatment efficacy among individuals with osteoarthritis.

There are numerous patient-reported outcome measures to assess individuals with osteoarthritis. Excessive proliferation of these measures and lack of standardization in their use, however, hinder comparisons across studies and populations [5]. Furthermore, using multiple measures may be time consuming for patients, cost prohibitive, and not practical for all settings. The National Institutes of Health's (NIH) Patient Reported Outcomes Measurement Information System (PROMIS) has introduced a number of static short-form patient-reported outcome measures, which offer an efficient and cost-effective alternative [6]. PROMIS is unique because it is intended for clinicians and researchers in various disciplines who are interested in measuring physical, mental, and social health among individuals with various chronic conditions. Several static-short form instruments target constructs relevant to patients with symptomatic knee osteoarthritis, including PROMIS Pain Interference 6b, Physical Function 10a, Emotional Distress-Anxiety 7a, and Emotional Distress-Depression 8b. Evidence for the validity of these instruments was initially obtained based on analysis of responses from a general population sample and from clinical samples, including a subset with osteoarthritis [7]. However, no prior study has evaluated the properties of PROMIS static short-form scores among individuals with symptomatic knee osteoarthritis.

Our objective was to evaluate the floor/ceiling effects and construct (structural) validity of four PROMIS ShortForm Instruments (i.e., Pain Interference 6b, Physical Function 10a, Emotional Distress-Anxiety 7a, and Emotional Distress-Depression 8b) function among a wellcharacterized sample of individuals with knee osteoarthritis. To achieve our goals we asked participants to complete six legacy measures: Medical Outcomes Short Form-36 (SF-36) [8-10], the Western Ontario and McMaster Universities Arthritis Index (WOMAC) [11-13], two objective physical function tests (i.e., 6-min walk test, 20-meter walk test) [14], the Perceived Stress Scale [15], and the Beck Depression Inventory Second Edition (BDI-II) [16-19]. These measures of pain, function, and psychological health domains were chosen because they relate to the constructs measured by the four PROMIS static short-form instruments and are widely used and well-validated among individuals with knee osteoarthritis.

\section{Methods}

We conducted a secondary analysis of baseline data obtained in our NIH-funded, randomized trial that compared Tai Chi and physical therapy among individuals with symptomatic knee osteoarthritis (Trial Registry \#NCT01258985) [20]. This secondary dataset is unique because it includes responses to a wide range of patient-reported outcome measures, both PROMIS measures and well-validated (legacy) measures used widely in studies of knee osteoarthritis. Data were collected at Tufts Medical Center, an urban tertiary care academic hospital in Boston, USA. We received ethics approval for this study from the Tufts Medical Center/ Tufts University Human Institutional Review Board. To be eligible, participants had to meet the following criteria: 1 ) age $\geq 40$ years; 2 ) Western Ontario and McMaster Universities Arthritis Index (WOMAC) pain subscale score (100 mm visual analog scales) $>40$ on at least 1 out of 5 questions; 3) fulfillment of the American College of Rheumatology criteria for knee osteoarthritis [21]; 4) radiographic evidence of knee osteoarthritis defined as the presence of osteophytes in the tibiofemoral and/or the patellofemoral compartment, as assessed on standing anterior-posterior and lateral views; and 5) confirmation of knee pain, discomfort, or disability by clinical examination. We excluded individuals who had experience in the past year with physical therapy, Tai Chi training, or similar types of alternative medicine (e.g., Qi Gong or yoga); serious medical conditions limiting their ability to fully participate as determined by a primary care physician; intra-articular steroid injections or replacement surgery on the affected knee in the previous three months; or a Mini-Mental examination score $<24$. All participants enrolled in the study provided informed consent. For this secondary analysis, we included all 204 participants who had their baseline visits between March 2011 and June 2013. These 204 participants were selected after staff prescreened almost 1,200 individuals on the phone and then screened approximately 280 individuals in person. The most common reason a person was not randomized into the study was if the individual failed to meet the inclusion criteria or an individual declined to participate.

\section{PROMIS instruments}

Participants enrolled in the trial completed four PROMIS static short-form, version 1.0 instruments including PROMIS Pain Interference 6b, Physical Function 10a, Emotional Distress-Anxiety 7a, and Emotional Distress-Depression 8b. These PROMIS instruments were the original versions of the short forms. The instruments were selected to meet the goal of the parent study: to compare the influence of Tai 
Chi or physical therapy on physical and psychological outcome measures.

The validity of PROMIS instruments has been assessed in both general and clinical U.S. sample populations [7, 22-24]. PROMIS short forms represent the items of the PROMIS item banks constructed to measure the targeted constructs. All of the included PROMIS short forms used 5-point Likert-type response categories to capture intensity, frequency, or duration, described in detail below. The instruments use a seven-day recall period, with the exception of PROMIS Physical Function, which does not reference any timeframe.

PROMIS instruments are publicly available on the PROMIS Assessment Center Library website: http://www.assessmentcenter.net/PromisForms.aspx. Sample questions are available at http://www.nihpromis.org/measures/SampleQuestions. Scoring manuals for PROMIS measures (http://www.assessmentcenter.net/Manuals.aspx) outline the development of the short forms (also see http:// www.nihpromis.org/science/sciencehome), report psychometric properties for each instrument, and describe how to identify PROMIS T-scores based on short form raw summed item scores. For all of the PROMIS short forms we reported the PROMIS T-scores.

PROMIS Short Form v1.0 - Pain Interference 6b measures "impact of pain on physical, mental, and social activities" (6). It consists of 6 items measured on a 5-point Likert-type scale (first 5 questions: "not at all" to "very much"; final question: "never" to "always"). The questions assess the degree to which pain interferes with enjoyment in life, ability to concentrate, day-to-day activities, recreational activities, tasks away from home, and socializing with others. The scores range from 41 to 78.3 with higher scores representing worse pain impact.

PROMIS Short Form v1.0 - Physical Function 10a measures "ability to carry out various activities that require physical capability, ranging from self-care to more vigorous activities that require increasing degrees of mobility, strength, or endurance" [7]. Half the 10 items of this form address the degree to which the respondent's health limits physical activities such as carrying groceries, climbing stairs, or participating in sports ("not at all" to "cannot do"). The other five items address the level of difficulty faced in carrying out activities of daily living such as vacuuming or tying one's shoelace ("without any difficulty" to "unable to do"). The scores range from 14.1 to 61.7 with higher scores representing better functioning.

PROMIS Short Form v1.0 - Emotional Distress- Anxiety 7a measures "fear, anxious misery, hyperarousal, and somatic symptoms related to arousal" [7]. The instrument consists of 7 items that ask respondents about the frequency with which they experienced emotions such as fear, stress, and anxiety ("never" to "always"). Scores range from 36.3 to 82.7 with higher scores indicating worse anxiety.
PROMIS Short Form v1.0 - Emotional Distress - Depression $8 \mathrm{~b}$ measures "negative mood, decrease in positive affect, information processing deficits, negative views of the self, and negative social cognition" [7]. It consists of 8 items in which respondents indicate the frequency with which they have experienced emotions such as worthlessness, hopelessness, and sadness ("never" to "always"). Scores range from 35.2 to 82.4 with higher scores representing worse depression.

\section{Legacy measures}

The SF-36 is a measure of general health status comprised of eight scales: physical functioning, social functioning, bodily pain, energy and vitality, mental health, role limitations due to physical problems, role limitations due to emotional problems, and general health. The eight scales are summarized in the Physical Component Summary (PCS) and Mental Component Summary (MCS) scores. The SF36 uses a 4-week recall period and consists of 36 items. Scores range from 0 to 100 with higher scores indicating better health. The SF-36 has been used widely in clinical osteoarthritis trials and the validity of SF-36 scores as a measure of health status has been well documented [8-10].

The WOMAC is a disease-specific measure designed to evaluate joint-specific health status and health outcomes in knee and hip osteoarthritis. We used the form consisting of $100 \mathrm{~mm}$ horizontal visual analogue scales with a 48-h recall period. It has three subscales: pain (score range, $0-500)$, stiffness $(0-200)$, and function (0-1700) with higher subscale scores indicating more severe disease. The validity of WOMAC has been well-established in knee and hip osteoarthritis [11-13].

The 6-min walk test and 20-m walk test are performancebased measures of gait velocity used to assess lower extremity function and mobility. The 6-min walk test is a measure of distance walked (measured in meters), while walking as fast as possible, in a total of six minutes, with greater distances indicating higher capacity. The 20-m walk test is a measure of time (measured in seconds) required to walk a total of twenty meters, with longer times indicating lower capacity. Both tests were performed in quiet hallways and were administered by trained investigators following a standard script. Gait velocity measures are valid for use in knee osteoarthritis populations [14].

The Perceived Stress Scale is a measure of non-specific stress appraisal. It uses a 1-month recall period and consists of 10 items on a 5-point Likert scale. Scores range from 0 to 40 with higher scores indicating higher levels of experienced stress. The validity of Perceived Stress Scale scores has been well documented in knee osteoarthritis studies [15].

The BDI-II is a measure of depression that assesses commonly associated signs and symptoms according the Diagnostic and Statistical Manual of Mental Disorders, 
Fourth Edition criteria. It uses a 2-week recall period and consists of 21 multiple-choice items. Scores range from 0 to 63 with higher scores indicating more severe depression. The BDI-II has been validated for use among non-psychiatric populations and has been used to assess depression in multiple knee osteoarthritis studies [16-19].

\section{Procedure}

As part of their baseline assessment and prior to any intervention, participants completed the outcome measures described above. The outcome measures were completed in the following order: WOMAC, SF-36, Perceived Stress Scale, BDI-II, PROMIS Pain Interference, PROMIS Physical Function, PROMIS Depression, and PROMIS Anxiety. All of the self-reported outcome measures were collected and managed using REDCap electronic data capture tools [25] hosted at Tufts Medical Center except for WOMAC, which was completed on paper. The two physical function tests were completed on site and were administered either prior to or after completion of the questionnaires, depending on the availability of research staff.

\section{Statistical analysis}

The analyses reported here was limited to baseline data obtained from participants that met eligibility criteria and were enrolled in the study. We calculated descriptive statistics and percentages of participants scoring the minimum (floor) and maximum (ceiling) possible scores. We defined important floor or ceiling effects as more than $15 \%$ of participants achieving the lowest or highest score, respectively [26]. Because not all data were normally distributed, we estimated the association between scores on the PROMIS instruments and legacy measures using the non-parametric, Spearman's rank correlation coefficient.

\section{Comparison of results with previous literature}

Tests of the statistical significance of differences in associations were outside the scope of this study and observed differences in point estimates of correlations should not be over-interpreted. However, to provide an interpretive context for our results, we presented the current results in the context of previous correlational studies. We conducted a comprehensive literature search (in Summer 2014) for articles that reported correlations between scores from legacy measures and PROMIS scores or other instruments that measure similar constructs. Results gleaned from published literature were grouped by clinical status: general population, osteoarthritis, other clinical conditions, and the current study results. Findings were plotted by condition on a graph to visually represent the range of findings in previous studies.

\section{Results}

\section{Demographic data and descriptive statistics}

Our analysis included data from 204 participants with an average age of 60 years and body mass index of $33 \mathrm{~kg} / \mathrm{m}^{2}$ (Table 1). Ninety-two percent had a Kellgren/ Lawrence grade $\geq 2$ in the tibiofemoral joint with a median self-reported duration of knee pain of 5 years. Table 1 includes additional demographic and clinical characteristics for the sample.

Floor or ceiling effects are reported in Table 2. Additional file 1: Table S1 and Figs. 1 and 2 report the correlation coefficients between PROMIS static short-form instruments and legacy measures.

\section{PROMIS pain interference}

PROMIS Pain Interference demonstrated minimal floor effect with only $5 \%$ of participants scoring the minimum PROMIS Pain Interference score. Pain Interference scores had stronger correlations with measures of whole body pain (SF-36 Bodily Pain, $r=-0.73,95 \%$ confidence interval $[\mathrm{CI}]=-0.79$ to -0.65 ) and physical health (SF-36 PCS, $r=-0.73,95 \% \mathrm{CI}=-0.79$ to -0.66$)$ than other legacy measures, including knee-specific pain (WOMAC pain, $r=0.47,95 \% \mathrm{CI}=0.35$ to 0.57 ) or gait speed (20$\mathrm{m}$ walk test, $r=0.34,95 \% \mathrm{CI}=0.21$ to 0.45 .

\section{PROMIS physical function}

PROMIS Physical Function scores had no floor effect since all of the participants scored above the minimum but one participant reported the highest possible score. PROMIS Physical Function scores had stronger correlations with measures of whole body function than with other legacy measures, including patient-reported lower extremity function or objective measures of lower extremity performance. Specifically, PROMIS Physical

Table 1 Demographic and clinical characteristics of patients with knee osteoarthritis $(n=204)$

\begin{tabular}{ll}
\hline Characteristic & $\begin{array}{l}\text { Distribution } \\
\mathrm{n}(\%)^{\mathrm{a}}\end{array}$ \\
\hline Age, mean \pm SD years & $60.2(10.5)$ \\
Body Mass Index, mean \pm SD $\mathrm{kg} / \mathrm{m}^{2}$ & $32.8(7.2)$ \\
$\begin{array}{l}\text { Self-reported duration of knee pain, median } \\
\left(25^{\text {th }}, 75^{\text {th }} \text { percentile) years }\right.\end{array}$ & $5.0(3.0,10.0)$ \\
Tibiofemoral Kellgren/Lawrence grade $\geq 2$ & $183(92.4)$ \\
Female Sex & $143(70.1)$ \\
Race & \\
$\quad$ White & $107(52.7)$ \\
Black & $72(35.5)$ \\
Other & $24(11.8)$ \\
Married & $52(25.5)$ \\
Greater than high school education & $170(83.3)$ \\
\hline
\end{tabular}

${ }^{a} \mathrm{n}(\%)$ unless noted otherwise. SD = standard deviation 
Table 2 Descriptive statistics of outcome measures in patients with knee osteoarthritis including floor and ceiling effects

\begin{tabular}{|c|c|c|c|c|c|c|c|c|}
\hline \multirow[t]{2}{*}{ Outcome Measure } & \multicolumn{4}{|l|}{ Scores } & \multicolumn{2}{|c|}{ Floor } & \multicolumn{2}{|c|}{ Ceiling } \\
\hline & Score Range & $n$ & Mean \pm SD & Median (range) & $n$ & $\%$ & $n$ & $\%$ \\
\hline PROMIS Pain Interference & $41-78.3$ & 204 & $58.0(7.0)$ & $58.1(41.0-74.4)$ & 11 & 5.4 & 0 & 0 \\
\hline PROMIS Physical Function ${ }^{a}$ & $14.1-61.7$ & 204 & $40.7(5.5)$ & $40.2(28.8-61.7)$ & 0 & 0 & 1 & 0.5 \\
\hline PROMIS Anxiety & $36.3-82.7$ & 203 & $50.2(8.9)$ & $51.3(36.3-72.9)$ & 34 & 16.8 & 0 & 0 \\
\hline PROMIS Depression & $37.1-81.1$ & 202 & $48.9(8.9)$ & $48.2(37.1-77.9)$ & 49 & 24.3 & 0 & 0 \\
\hline SF-36 PCS ${ }^{a}$ & $0-100$ & 204 & $36.6(9.1)$ & $36.7(14.0-59.8)$ & 0 & 0 & 0 & 0 \\
\hline SF-36 MCS ${ }^{a}$ & $0-100$ & 204 & $52.5(9.2)$ & $54.5(21.6-68.1)$ & 0 & 0 & 0 & 0 \\
\hline SF-36 Bodily Pain ${ }^{a}$ & $0-100$ & 204 & $47.5(18.6)$ & $41.0(0.0-100.0)$ & 1 & 0.5 & 4 & 2.0 \\
\hline SF-36 Physical Function & $0-100$ & 204 & $52.0(22.2)$ & $50.0(0.0-100.0)$ & 2 & 1.0 & 3 & 1.5 \\
\hline WOMAC Pain & $0-500$ & 203 & $254(98.6)$ & $239.8(50.7-500.0)$ & $\mathrm{n} / \mathrm{a}$ & $\mathrm{n} / \mathrm{a}$ & 1 & 0.5 \\
\hline WOMAC Function & $0-1700$ & 204 & $899.0(352.4)$ & $906.7(218.6,1700.0)$ & 0 & 0 & 1 & 0.5 \\
\hline 6-min Walk Test ${ }^{\mathrm{ab}}$ (meters) & $\mathrm{n} / \mathrm{a}$ & 199 & $395.5(90.1)$ & $392.8(89.1-645.3)$ & $\mathrm{n} / \mathrm{a}$ & $\mathrm{n} / \mathrm{a}$ & $\mathrm{n} / \mathrm{a}$ & $n / a$ \\
\hline 20-Meter Walk Test ${ }^{a}$ (seconds) & $\mathrm{n} / \mathrm{a}$ & 202 & $19.0(5.3)$ & $18.2(9.6-66.8)$ & $\mathrm{n} / \mathrm{a}$ & $\mathrm{n} / \mathrm{a}$ & $\mathrm{n} / \mathrm{a}$ & $\mathrm{n} / \mathrm{a}$ \\
\hline PSS & $0-40$ & 203 & $13.3(7.0)$ & $13.0(0.0,37.0)$ & 5 & 2.5 & 0 & 0 \\
\hline BDI-\| & $0-63$ & 199 & $7.6(8.6)$ & $5.0(0.0-39.0)$ & 27 & 13.6 & 0 & 0 \\
\hline
\end{tabular}

PROMIS = Patient Reported Outcomes Measurement Information System (reported as T-scores); SF-36= Medical Outcomes Short Form-36, MCS = Mental Component Summary, $P C S=$ Physical Component Summary, $B P=$ Bodily Pain; WOMAC = Western Ontario and McMaster Universities Arthritic Index; $P S S=$ Perceived Stress Scale; $B D I-I I=$ Beck Depression Index II

${ }^{a}$ Higher scores indicate better health-related outcomes (for other measures, higher scores indicates worse health-related outcomes)

${ }^{b}$ Does not include patients who refused, or attempted and failed

Function scores correlated well with SF-36 Physical Function ( $r=0.79,95 \% \mathrm{CI}=0.73$ to 0.84$)$ compared with WOMAC function $(r=-0.48,95 \% \mathrm{CI}=-0.58$ to -0.36), gait speed $(r=-0.43,95 \% \mathrm{CI}=-0.53$ to -0.31$)$, or 6-min walk times $(r=0.46,95 \% \mathrm{CI}=0.34$ to 0.56$)$.

\section{PROMIS anxiety}

PROMIS Anxiety scores had a floor effect; $17 \%$ of participants scored the minimum. For comparison, $3 \%$ of participants had a minimum Perceived Stress Scale score. PROMIS Anxiety scores had stronger correlations with measures of stress $(r=0.71,95 \% \mathrm{CI}=0.64$ to 0.77$)$ and depression $(r=0.70,95 \% \mathrm{CI}=0.62$ to 0.76$)$ than other legacy measures. For example, PROMIS Anxiety scores had small to moderate correlations with measures of function $(r=-0.40$ to 0.17$)$ and pain $(r=-0.41$ and 0.20$)$.

\section{PROMIS depression}

PROMIS Depression scores also had a floor effect; $24 \%$ of participants scored the minimum. For comparison, $14 \%$ of participants had a minimum BDI-II score. PROMIS Depression scores had stronger correlations with measures of stress $(r=0.65,95 \% \mathrm{CI}=0.56$ to 0.72$)$ and depression $(r=0.70,95 \% \mathrm{CI}=0.62$ to 0.76$)$ than with other legacy measures. For example, PROMIS Depression scores had small to moderate correlations with measures of physical function $(r=-0.31$ to 0.11$)$ and pain (SF-36 Bodily Pain: $r=-0.31$ and WOMAC knee pain: $r=0.19)$.

\section{Comparison to previous literature}

Figure 1 (PROMIS Pain Interference and Physical Function) and Fig. 2 (PROMIS Depression and Anxiety) visually locate the current study results in the range of findings from previous studies. In general, the values obtained in the current study were similar both to those obtained in other studies of individuals with osteoarthritis and in studies using other samples. As already noted, tests of the statistical significance of differences in associations were outside the scope of this study, and observed differences in point estimates should not be over-interpreted. We did note some putative differences that could warrant additional study (see Additional file 1: Table S1, Figs. 1 and 2). Pairs of measures that generated lower correlation estimates in the current study compared to previous studies included: a) PROMIS Pain Interference scores and SF-36 Bodily Pain scores, and b) PROMIS Physical Function scores with the WOMAC Pain, WOMAC Function, SF-36 Physical Function, and gait speed. Pairs of measures that generated higher correlation estimates in the current study compared to previous studies included: a) PROMIS Pain Interference scores and scores on patientreported mental health scores (e.g., SF-36 MCS, BDI-II) and b) PROMIS Physical Function scores on the same measures.

\section{Discussion}

PROMIS is a novel system of free instruments that clinicians and researchers in various medical disciplines can use. The PROMIS static short-forms offer an efficient and 


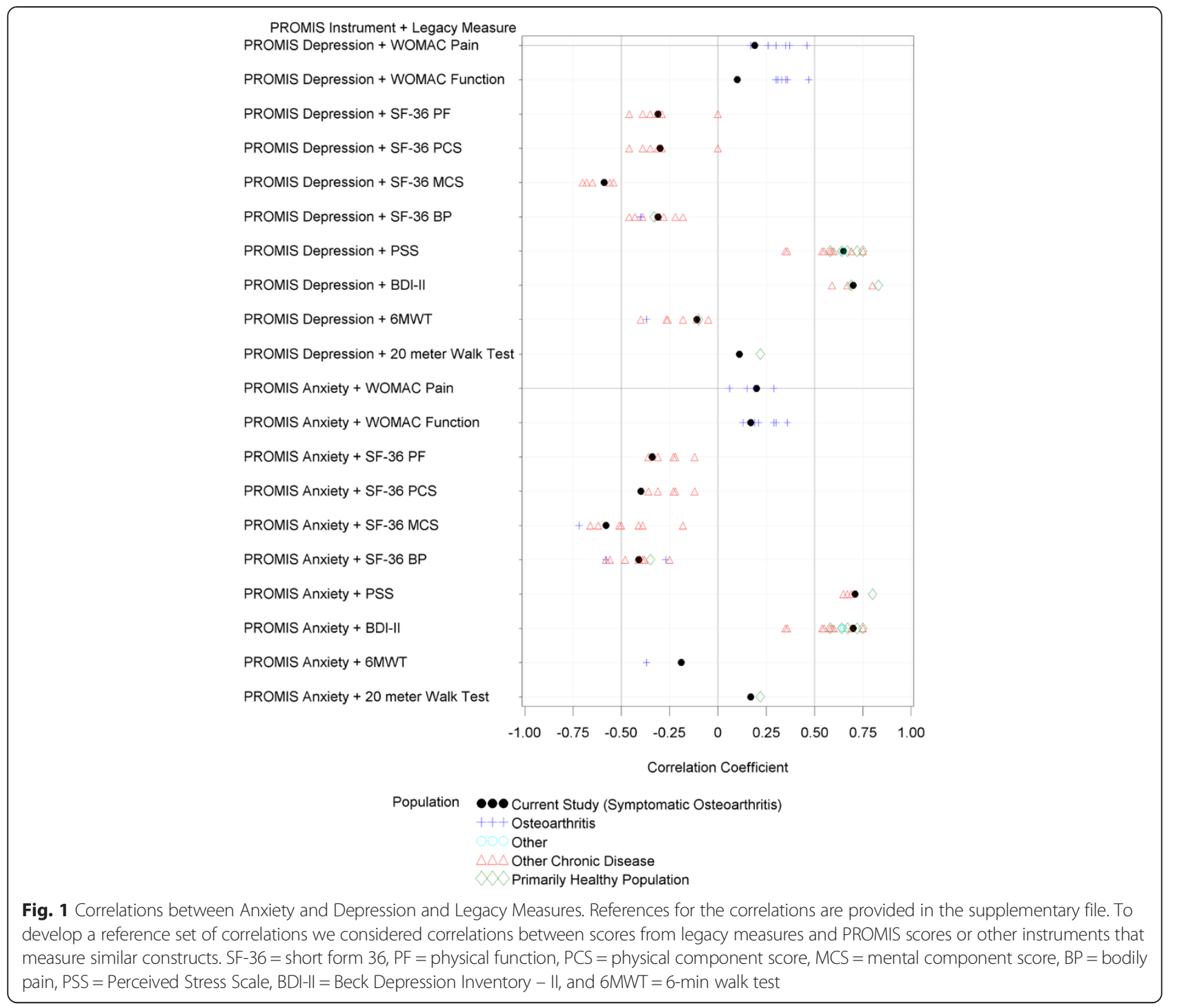

cost-effective means of measuring patient-reported outcomes critical to the assessment of knee osteoarthritis. This is the first study to evaluate the performance of the PROMIS static short-form instruments specifically among participants with symptomatic knee osteoarthritis. We found that PROMIS Anxiety and Depression measure a similar construct to other patient-reported outcomes that assess general mental health, including stress and depression, among patients with symptomatic knee osteoarthritis. The PROMIS Anxiety and Depression had a floor effect but the implications of this for future studies depend on study goals. For clinicians and researchers interested in distinguishing among individuals with low levels of anxiety or depression, the PROMIS short forms would not be a good choice. For example, if a researcher wished to explore an intervention's impact on depression or anxiety by investigating whether it would benefit individuals with anxiety below the US mean then the short forms may not be ideal. The floor effect could impede detection of a treatment effect. However, these floor effects might be unimportant in a study of a clinical population in which the presence of anxiety and depression has been documented.

The associations we found between scores on PROMIS measures and legacy measures suggest that the PROMIS Pain Interference and Physical Function scores target whole body pain and physical function among individuals with symptomatic knee osteoarthritis. Recently, Broderick et al. demonstrated known-groups validity (a subtype of construct validity) of the computer adaptive administrations of PROMIS Pain Interference and Physical Function in a study comparing patients with osteoarthritis to the general population [27]. Our results complement and expand upon those of Broderick et al., specifically among individuals with symptomatic knee osteoarthritis. Our findings indicate that PROMIS Pain Interference and Physical Function have stronger correlations with assessments of 


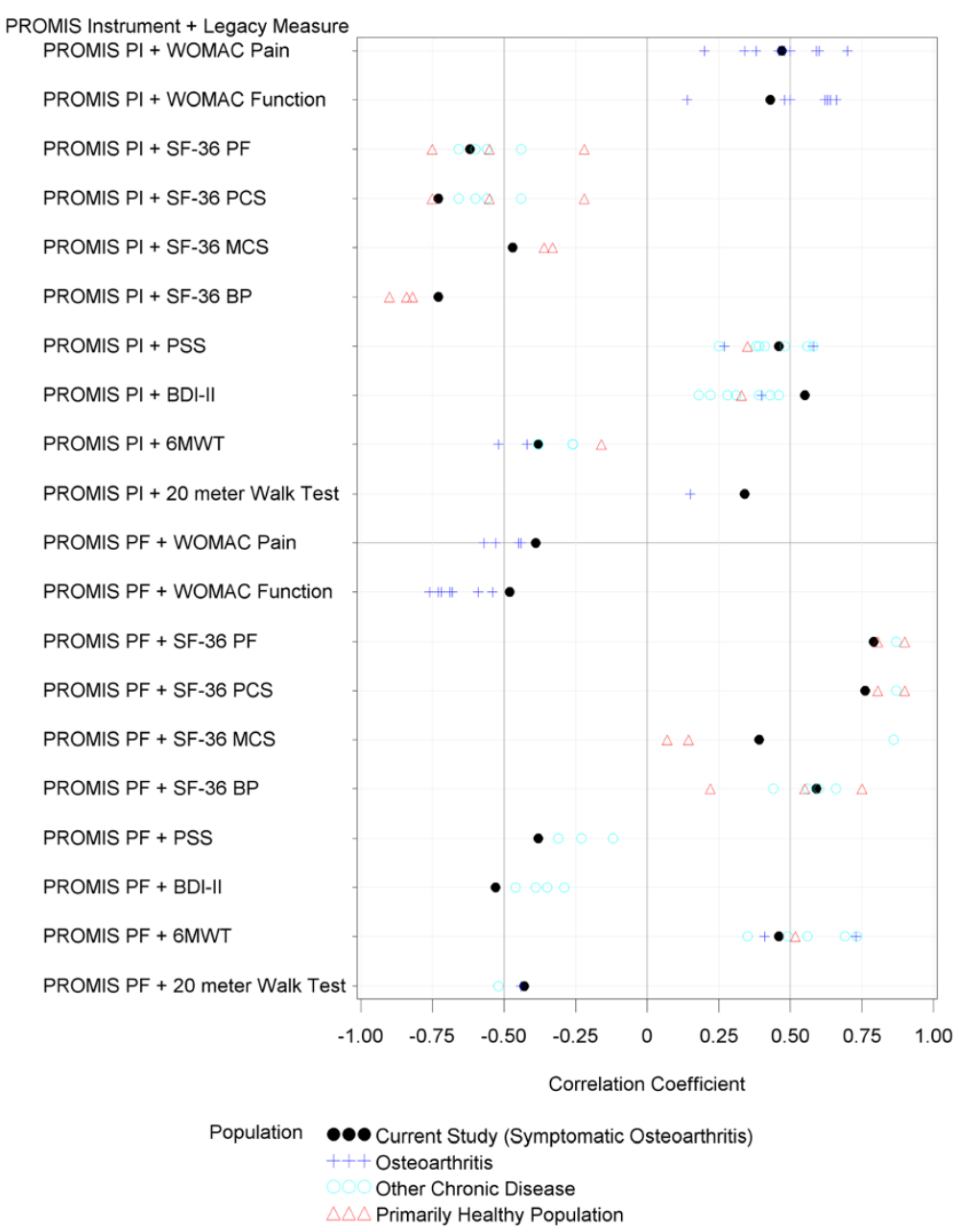

Fig. 2 Correlations between Pain Interference (PI) and Physical Function (PF) and Legacy Measures. References for the correlations are provided in the supplementary file. To develop a reference set of correlations we considered correlations between scores from legacy measures and PROMIS scores or other instruments that measure similar constructs. SF-36 = short form 36, PF = physical function, PCS = physical component score, MCS $=$ mental component score, BP = bodily pain, PSS = Perceived Stress Scale, BDI-II = Beck Depression Inventory $-\|$, and $6 \mathrm{MWT}=6-\mathrm{min}$ walk test

whole-body disease burden (i.e., SF-36 Bodily Pain, SF-36 PCS) compared to joint-specific measures (i.e., WOMAC pain, WOMAC function, and physical function tests). Conceptually, this is expected since the two PROMIS static short-form instruments include questions that are not lower-extremity specific.

During the initial item-evaluation process of the full PROMIS Physical Function item bank, the data satisfied criteria for unidimensionality, suggesting that the four subdomains of mobility (lower extremity), dexterity (upper extremity), axial or central (neck or back), and complex activities could be collapsed into one domain [28]. Bruce et al. pointed out however, that the analysis was conducted among individuals with relatively little disease burden compared with the typical clinical trial participant, raising the question of whether the unidimensional model can be applied among other populations. After the data used in the current study were collected,
Hays, et al. [29] identified upper-extremity and mobility subdomains of PROMIS Physical Function data. The two subdomains shared $35 \%$ of the variance in common. Subsets of items targeting these two subdomains were identified and scored using PROMIS parameters. This work allows measurement of upper-extremity function and mobility as well as overall physical functioning within the PROMIS system. Clinicians and researchers, who are interested in the local effects of knee osteoarthritis, may prefer using PROMIS lower-extremity items [29] or a joint-specific pain and function outcome (e.g., WOMAC). However, if the goal is to assess whole body pain or function (e.g., assessing an exercise intervention among patients with osteoarthritis at the knee and other joints) then the short form may be appropriate. Ultimately, many investigators or clinicians may want to consider lowerextremity specific outcomes and whole body changes. Therefore, using a computer adaptive version of PROMIS 
or a larger set of the PROMIS items may enable the simultaneous assessment of whole-body and lower-extremity specific outcomes, which would reduce the number of outcome instruments a participants needs to complete and reduce study expenses associated with paying for multiple patient-reported outcomes.

Our study is limited. Our visual representation of correlation results from the current study and previous research highlighted some observable differences in point estimates of associations. It was outside the scope of this study to evaluate whether these putative differences were significantly different or accountable to random variation. First, because the order of outcome measure administration was consistent across participants, order bias and patient fatigue may have affected patients' responses to self-report measures and performance on physical function tests. Second, our analyses did not include patients who refused or attempted and failed the two physical function tests $(6$-min walk test $[n=4] ; 20$-m walk test $[n=2])$. Because patients who attempted and failed the physical function tests represent the extremes of functional impairment, our analysis overestimates the overall function of our cohort. However, this subgroup represents a small proportion of our overall sample and thus we did not feel this significantly influenced our overall results. Third, our definition of floor and ceiling effects was likely a conservative estimate. We measured the number of patients with the minimum and maximum scores rather than clinically-defined scores with thresholds representative of the extremes of health-related outcomes. Longitudinal studies will be needed to assess the minimally important change to better estimate the floor and ceiling effects.

More work needs to be done to understand the role of PROMIS static short-form instruments in evaluating patients with knee osteoarthritis. Future studies might assess time savings and cost efficiency, as well as other key psychometric properties as outlined by the Consensusbased Standards for the Selection of Health Measurement Instruments (COSMIN) [30]; including, responsiveness of scores on PROMIS short-forms in detecting change and score interpretability among patients with knee osteoarthritis. Lastly, computerized-adaptive administrations of PROMIS instruments should also be tested among patients with knee osteoarthritis.

\section{Conclusions}

In conclusion, scores on the four tested PROMIS static short forms demonstrated good performance among patients with symptomatic knee osteoarthritis as evidenced in correlations with legacy measures. The patterns of correlations suggest that PROMIS Anxiety and Depression targets general mental health (e.g., stress, depression), and PROMIS Pain Interference and Physical Function static short forms target whole body outcomes (e.g., SF-36) among participants with symptomatic knee osteoarthritis. These instruments may be useful in clinical research among individuals with symptomatic knee osteoarthritis. However, floor effects in the PROMIS Anxiety and Depression scores should be considered if needing to distinguish among patients with very low levels of these outcomes.

\section{Additional file}

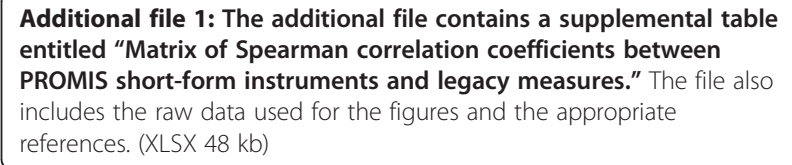
entitled "Matrix of Spearman correlation coefficients between PROMIS short-form instruments and legacy measures." The file also includes the raw data used for the figures and the appropriate references. (XLSX $48 \mathrm{~kb}$ )

\section{Abbreviations}

PROMIS: Patient Reported Outcomes Measurement Information System; WOMAC: Western Ontario and McMaster Universities Arthritis Index; SF-36: Short form-36; BDI-II: Beck Depression Inventory Second Edition; PCS: Physical Component Summary; MCS: Mental Component Summary.

\section{Competing interests}

The authors declare that they have no competing interests.

\section{Authors' contributions}

JBD contributed to the conception and design, acquisition of data, analysis, and interpretation of data, drafting/revisions of article, as well as final approval of the article. NM contributed to the conception and design, acquisition of data, analysis, and interpretation of data, drafting/revisions of article, as well as final approval of the article. LLP contributed to the conception and design, acquisition of data, analysis, and interpretation of data, drafting/revisions of article, as well as final approval of the article. KFC contributed to the conception and design, interpretation of data, drafting/ revisions of article, as well as final approval of the article. CCW contributed to the conception and design, acquisition of data, analysis, and interpretation of data, drafting/revisions of article, as well as final approval of the article.

\section{Acknowledgements}

This study was funded by the National Center for Complementary and Alternative Medicine (NCCIH; Grant \# R01AT005521-01A1, NCT01258985, $\mathrm{PI}$ - Dr. Chenchen Wang).

\section{Author details}

'Division of Rheumatology, Tufts Medical Center, 800 Washington Street, Box \#406, Boston, MA 02111, USA. ${ }^{2}$ Internal Medicine Residency Program, John A Burns School of Medicine, University of Hawaii, Honolulu, HI, USA. ${ }^{3}$ The Institute for Clinical Research and Health Policy Studies, Tufts Medical Center, and Tufts Clinical and Translational Science Institute, Tufts University, 800 Washington Street, Box \#63, Boston, MA 02111, USA. ${ }^{4}$ Department of Medical Social Sciences, Feinberg School of Medicine, Northwestern University, Chicago, IL, USA.

Received: 6 March 2015 Accepted: 9 September 2015 Published online: 14 September 2015

\section{References}

1. Lane NE, Brandt K, Hawker G, Peeva E, Schreyer E, Tsuji W, et al. OARSI-FDA initiative: defining the disease state of osteoarthritis. Osteoarthritis Cartilage. 2011;19:478-82.

2. Hawker GA, French MR, Waugh EJ, Gignac MA, Cheung C, Murray BJ. The multidimensionality of sleep quality and its relationship to fatigue in older adults with painful osteoarthritis. Osteoarthritis Cartilage. 2010;18:1365-71.

3. Hawker GA, Gignac MA, Badley E, Davis AM, French MR, Li Y, et al. A longitudinal study to explain the pain-depression link in older adults with osteoarthritis. Arthritis Care Res. 2011;63:1382-90. 
4. Phillips K, Clauw DJ. Central pain mechanisms in the rheumatic diseases: future directions. Arthritis Rheum. 2013;65:291-302.

5. Fries JF, Krishnan E, Bruce B. Items, Instruments, Crosswalks, and PROMIS. J Rheumatol. 2009;36:1093-5.

6. Khanna D, Krishnan E, Dewitt EM, Khanna PP, Spiegel B, Hays RD. The future of measuring patient-reported outcomes in rheumatology: Patient-Reported Outcomes Measurement Information System (PROMIS). Arthritis Care Res. 2011;63 Suppl 11:S486-90.

7. Cella D, Riley W, Stone A, Rothrock N, Reeve B, Yount S, et al. The PatientReported Outcomes Measurement Information System (PROMIS) developed and tested its first wave of adult self-reported health outcome item banks: 2005-2008. J Clin Epidemiol. 2010;63:1179-94.

8. Ware Jr JE, Sherbourne CD. The MOS 36-item short-form health survey (SF-36) I. Conceptual framework and item selection. Med Care. 1992;30:473-83.

9. Kosinski M, Keller SD, Ware Jr JE, Hatoum HT, Kong SX. The SF-36 Health Survey as a generic outcome measure in clinical trials of patients with osteoarthritis and rheumatoid arthritis: relative validity of scales in relation to clinical measures of arthritis severity. Med Care. 1999;37:Ms23-39.

10. Ware JE, Kosinski M, Dewey JE, Gandek B. SF-36 Health Survey: Manual and Interpretation Guide. 2000.

11. Bellamy N, Buchanan WW, Goldsmith CH, Campbell J, Stitt LW. Validation study of WOMAC: a health status instrument for measuring clinically important patient relevant outcomes to antirheumatic drug therapy in patients with osteoarthritis of the hip or knee. J Rheumatol. 1988;15:1833-40.

12. Bellamy N. WOMAC Osteoarthritis Index User's Guide. Version VII, Australia. 2005.

13. Wolfe F, Kong SX. Rasch analysis of the Western Ontario MacMaster questionnaire (WOMAC) in 2205 patients with osteoarthritis, rheumatoid arthritis, and fibromyalgia. Ann Rheum Dis. 1999;58:563-8.

14. Marks R. Reliability and validity of self-paced walking time measures for knee osteoarthritis. Arthritis Care Res. 1994;7:50-3.

15. Cohen S, Kamarck T, Mermelstein R. A global measure of perceived stress. J Health Soc Behav. 1983;24:385-96.

16. Beck AT, Ward CH, Mendelson M, Mock J, Erbaugh J. An inventory for measuring depression. Arch Gen Psychiatry. 1961;4:561-71.

17. Beck AT, Steer RA. Manual for the Beck Depression Inventory - Second Edition Manual. Texas: The Psychological Corporation; 1996.

18. Creamer P, Lethbridge-Cejku M, Hochberg MC. Factors associated with functional impairment in symptomatic knee osteoarthritis. Rheumatology (Oxford, England). 2000:39:490-6.

19. Summers MN, Haley WE, Reveille JD, Alarcon GS. Radiographic assessment and psychologic variables as predictors of pain and functional impairment in osteoarthritis of the knee or hip. Arthritis Rheum. 1988;31:204-9.

20. Wang C, Iversen MD, McAlindon TE, Harvey WF, Wong JB, Fielding RA, et al. Assessing the comparative effectiveness of tai chi versus physical therapy for knee osteoarthritis: design and rationale for a randomized trial. BMC Complement Altern Med 2014. In Press.

21. Altman R, Asch E, Bloch D, Bole G, Borenstein D, Brandt $K$, et al. Development of criteria for the classification and reporting of osteoarthritis. Classification of osteoarthritis of the knee. Diagnostic and Therapeutic Criteria Committee of the American Rheumatism Association. Arthritis Rheum. 1986;29:1039-49.

22. Amtmann D, Cook KF, Jensen MP, Chen WH, Choi S, Revicki D, et al Development of a PROMIS item bank to measure pain interference. Pain. 2010;150:173-82.

23. Bruce B, Fries J, Lingala B, Hussain YN, Krishnan E. Development and assessment of floor and ceiling items for the PROMIS physical function item bank. Arthritis Res Ther. 2013;15:R144.

24. Pilkonis PA, Choi SW, Reise SP, Stover AM, Riley WT, Cella D, et al. Item Banks for Measuring Emotional Distress From the Patient-Reported Outcomes Measurement Information System (PROMIS ${ }^{\circledR}$ ): Depression, Anxiety, and Anger. Assessment. 2011;18:263-83.

25. Harris PA, Taylor R, Thielke R, Payne J, Gonzalez N, Conde JG. Research electronic data capture (REDCap)-a metadata-driven methodology and workflow process for providing translational research informatics support. J Biomed Inform. 2009;42:377-81.

26. Terwee CB, Bot SD, de Boer MR, van der Windt DA, Knol DL, Dekker J, et al. Quality criteria were proposed for measurement properties of health status questionnaires. J Clin Epidemiol. 2007;60:34-42.

27. Broderick JE, Schneider S, Junghaenel DU, Schwartz JE, Stone AA. Validity and reliability of patient-reported outcomes measurement information system (PROMIS) instruments in osteoarthritis. Arthritis Care Res. 2013;65:1625-33.

28. Bruce B, Fries JF, Ambrosini D, Lingala B, Gandek B, Rose M, et al. Better assessment of physical function: item improvement is neglected but essential. Arthritis Res Ther. 2009;11:R191.

29. Hays RD, Spritzer KL, Amtmann D, Lai JS, Dewitt EM, Rothrock N, et al. Upper-extremity and mobility subdomains from the Patient-Reported Outcomes Measurement Information System (PROMIS) adult physical functioning item bank. Arch Phys Med Rehabil. 2013;94:2291-6.

30. Mokkink L, Terwee C, Patrick D, Alonso J, Stratford P, Knol D, et al. The COSMIN checklist for assessing the methodological quality of studies on measurement properties of health status measurement instruments: an international Delphi study. Qual Life Res. 2010;19:539-49.

\section{Submit your next manuscript to BioMed Central and take full advantage of:}

- Convenient online submission

- Thorough peer review

- No space constraints or color figure charges

- Immediate publication on acceptance

- Inclusion in PubMed, CAS, Scopus and Google Scholar

- Research which is freely available for redistribution 